\title{
Tight Bounds for Conflict-Free Chromatic Guarding of Orthogonal Art Galleries
}

\author{
Frank Hoffmann ${ }^{1}$, Klaus Kriegel ${ }^{1}$, Subhash Suri ${ }^{2}$, Kevin Verbeek $^{3}$, \\ and Max Willert ${ }^{1}$
}

1 Freie Universität Berlin, Institut für Informatik, 14195 Berlin, Germany

\{hoffmann, kriegel, willerma\}@mi.fu-berlin.de

2 Dept. of Computer Science, University of California, Santa Barbara, USA

suri@cs.ucsb.edu

3 Dept. of Mathematics and Computer Science, TU Eindhoven, The Netherlands

k.a.b.verbeek@tue.nl

\begin{abstract}
The chromatic art gallery problem asks for the minimum number of "colors" $t$ so that a collection of point guards, each assigned one of the $t$ colors, can see the entire polygon subject to some conditions on the colors visible to each point. In this paper, we explore this problem for orthogonal polygons using orthogonal visibility - two points $p$ and $q$ are mutually visible if the smallest axisaligned rectangle containing them lies within the polygon. Our main result establishes that for a conflict-free guarding of an orthogonal $n$-gon, in which at least one of the colors seen by every point is unique, the number of colors is $\Theta(\log \log n)$. By contrast, the best upper bound for orthogonal polygons under standard (non-orthogonal) visibility is $O(\log n)$ colors. We also show that the number of colors needed for strong guarding of simple orthogonal polygons, where all the colors visible to a point are unique, is $\Theta(\log n)$. Finally, our techniques also help us establish the first non-trivial lower bound of $\Omega(\log \log n / \log \log \log n)$ for conflict-free guarding under standard visibility. To this end we introduce and utilize a novel discrete combinatorial structure called multicolor tableau.
\end{abstract}

1998 ACM Subject Classification F.2.2 Nonnumerical Algorithms, G.2.2 Graph Theory

Keywords and phrases Orthogonal polygons, art gallery problem, hypergraph coloring

Digital Object Identifier 10.4230/LIPIcs.SOCG.2015.421

\section{Introduction}

The classic Art Gallery Problem (AGP) posed by Klee in 1973 asks for the minimum number of guards sufficient to watch an art gallery modelled by an $n$-sided simple polygon $P$. A guard sees a point in $P$ if the connecting line segment is contained in $P$. Therefore, a guard watches a star polygon contained in $P$ and the question is to cover $P$ by a collection of stars with smallest possible cardinality. The answer is $\left\lfloor\frac{n}{3}\right\rfloor$ as shown by Chvátal [3]. This result was the starting point for a rich body of research about algorithms, complexity and combinatorial aspects for many variants of the original question. Surveys can be found in the seminal monograph by O'Rourke [10], in Shermer [12], and Urrutia [15].

Graph coloring arguments have been frequently used for proving worst case combinatorial bounds for art gallery type questions starting with Fisk's proof [5]. Somehow surprisingly, chromatic versions of the AGP have been proposed and studied only recently. There are two chromatic variants: strong chromatic guarding and conflict-free guarding of a polygon $P$. In both versions we look for a guard set $G$ and give each guard one of $t$ colors. The chromatic

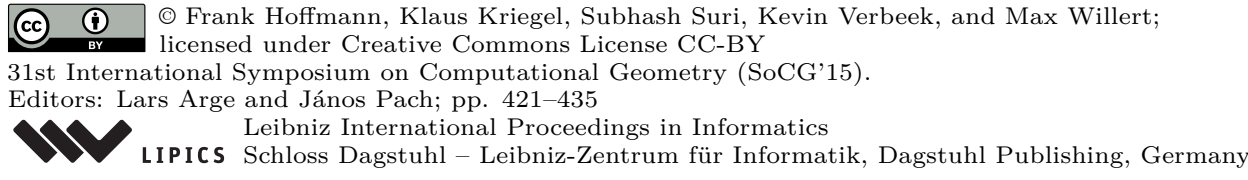



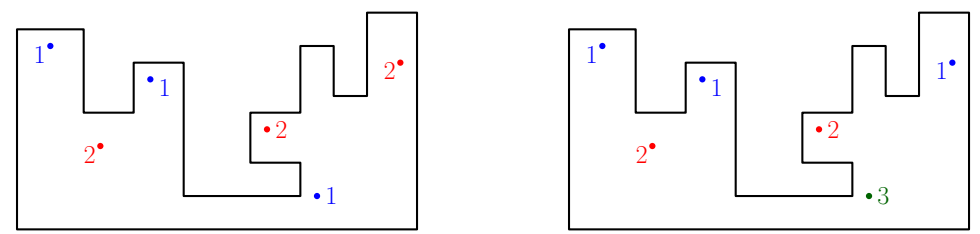

Figure 1 Example of conflict-free (left) and strong chromatic (right) r-guarding.

guarding is said to be strong if for each point $p \in P$ all guards $G(p)$ that see $p$ have pairwise different colors [4]. It is conflict-free if in each $G(p)$ there is at least one guard with a unique color, see [1]. The goal is to determine guard sets such that the number of colors sufficient for these purposes is minimal. Observe, in both versions minimizing the number of guards is not part of the objective function. Figure 1 shows a simple orthogonal polygon with both conflict-free and strong chromatic guardings in the orthogonal visibility model.

To grasp the nature of the problem, observe that it has two conflicting aspects. We have to guard the polygon but at the same time we want the guards to hide from each other, since then we can give them the same color. For example, in the strong version we want a guard set that can be partitioned into a minimal number of subsets and in each subset the pairwise orthogonal link distance is at least 3. Moreover, we will see a strong dependence of the results on the underlying visibility model, standard vs. orthogonal. We refer to standard and orthogonal visibility as l-visibility (line visibility) and r-visibility, respectively. We use superscripts $l$ and $r$ in the bounds to indicate the model.

Let $\chi_{s t}^{l}(n)$ and $\chi_{c f}^{l}(n)$ denote the minimal number of colors sufficient for any simple polygon on $n$ vertices in the strong chromatic and in the conflict-free version if based on line visibility.

Here is a short summary of known bounds. For simple orthogonal polygons on $n$ vertices $\chi_{c f}^{l}(n) \in O(\log n)$, as shown in [1]. The same bound applies to simple general polygons, see [2]. Both proofs are based on subdividing the polygon into weak visibility subpolygons that are in a certain sense independent with respect to conflict-free chromatic guarding. For the strong chromatic version we have $\chi_{s t}^{l}(n) \in \Theta(n)$ for simple polygons and $\chi_{s t}^{l}(n) \in \Omega(\sqrt{n})$ even for the monotone orthogonal case, see [4]. NP-hardness is discussed in [6]. In [4], simple $O(1)$ upper bounds are shown for special polygon classes like spiral polygons and orthogonal staircase polygons combined with line visibility.

Next we state our main contributions for simple orthogonal polygons:

1. For the strong chromatic version we show $\chi_{s t}^{r}(n) \in \Theta(\log n)$.

2. For the conflict-free chromatic version we show $\chi_{c f}^{r}(n) \in \Theta(\log \log n)$.

3. For line visibility guards we have: $\chi_{c f}^{l}(n) \in \Omega(\log \log n / \log \log \log n)$.

This is the first super-constant lower bound also for general simple polygons.

The chromatic AGP versions can be easily interpreted as coloring questions for concrete geometric hypergraphs. Smorodinsky ([14]) gives a nice survey of both practical and theoretical aspects of hypergraph coloring. A special role play hypergraphs that arise in geometry. For example, given a set of points $P$ in the plane and a set of regions $\mathcal{R}$ (e.g. rectangles, disks), we can define the hypergraph $H_{\mathcal{R}}(P)=(P,\{P \cap S \mid S \in \mathcal{R}\})$. The discrete interval hypergraph $H_{\mathcal{I}}$ is a concrete example of such a hypergraph: We take $n$ points on a line and all possible intervals as regions. It is not difficult to see that $\chi_{c f}\left(H_{\mathcal{I}}\right) \in \Theta(\log n)$. As to our AGP versions, we can associate with a given polygon and a guard set a geometric hypergraph. Its vertices are the guards and a hyperedge is defined by a set of guards for which there exists a point that can see exactly these guards. Then one wants to color this hypergraph in 
a conflict-free or in a strong manner. Another example is the following rectangle hypergraph. The vertex set is a set of $n$ axis-aligned rectangles and each maximal subset of rectangles with a common intersection forms a hyperedge. Here the order for the conflict-free chromatic number is $\Omega(\log n)$ and $O\left(\log ^{2} n\right)$ as shown in $[11,14]$.

Looking at our results, it is not a big surprise that the combination of orthogonal polygons with r-visibility yields the strongest bounds. This is simply due to additional structural properties and this phenomenon has already been observed for the original AGP. For example, the $\left\lfloor\frac{n}{4}\right\rfloor$ tight worst case bound for covering simple orthogonal polygons with general stars can also be proven for r-stars (see [10]) and it holds even for orthogonal polygons with holes, see [7]. Further, while minimizing the number of guards is NP-hard both for simple general and orthogonal polygons if based on line visibility, it becomes polynomially solvable for r-visibility in the simple orthogonal case, see $[9,17]$. The latter result is based on the solution of the strong perfect graph conjecture.

The paper is organized as follows. We give necessary basic definitions in the next section. Then we prove upper bounds in Section 3 using techniques developed in $[1,2]$. That means we also subdivide a simple orthogonal polygon into histograms which are independent with respect to chromatic guarding. To deal with a single histogram we introduce the notion of its spine tree. The spine tree provides an elegant and efficient way to describe r-visibility properties of the histogram. Our main contributions are the lower bound proofs in Section 4. Especially, we introduce a novel combinatorial structure called multicolor tableau. This structure enables us to show a first super-constant lower bound for chromatic conflict-free guarding based on the line visibility model.

\section{Preliminaries}

We study simple orthogonal polygons, i.e., polygons consisting of alternating vertical and horizontal edges only. By $|P|$ we denote the number of vertices, by $\partial P$ the boundary and by $\operatorname{int} P=P \backslash \partial P$ the interior of the polygon. Vertices can be reflex or convex. A reflex vertex has an interior angle $3 \pi / 2$ while convex vertices have an interior angle of $\pi / 2$. We do not make any general position assumption for the simple orthogonal polygons $P$. Points $p, q \in P$ are $r$-visible to each other if the closed axis-parallel rectangle $r[p, q]$ with diagonal $p q$ is contained in $P$. In the following, unless stated otherwise, visible always means rvisible. The visibility polygon of $p$, the set of all points visible from $p$, is formally defined as $V(p)=\{q \in P \mid r[p, q] \subseteq P\}$. A polygon that is fully visible from one of its points is called a star. For $P^{\prime} \subset P$ we define its visibility polygon by $V\left(P^{\prime}\right)=\cup_{p \in P^{\prime}} V(p)$. The windows of a subpolygon $P^{\prime}$ in $P$ are those parts of $\partial P^{\prime}$ that do not belong to $\partial P$.

For an orthogonal polygon $P$ we construct its induced $r$-visibility arrangement $\mathcal{A}^{r}(P)$ : For each reflex vertex of $P$ we extend both incident boundary edges into int $P$ until they meet the boundary again, therefore defining a subdivision of the polygon. The 2-dimensional faces of this arrangement are rectangles. Clearly, points from the interior of the same rectangle (subsequently called cell) have the same visibility polygon.

Finally, we define special classes of orthogonal polygons. A weak visibility polygon, also known as histogram, has a boundary base edge e connecting two convex vertices such that $V(e)=P$. A histogram that is a star is called a pyramid.

\section{Conflict-free and strong chromatic guarding}

A set $G$ of points is a guard set for an orthogonal polygon $P$ if their visibility polygons jointly cover the whole polygon. If in addition each guard $g \in G$ is assigned one color $\gamma(g)$ from a 
fixed finite set of colors $[t]=\{1,2, \ldots, t\}$ we have a chromatic guarding $(G, \gamma)$. Next we give the central definitions. Since these definitions are independent of the visibility model, we drop the superscripts $l$ and $r$ in the following.

A chromatic guard set $(G, \gamma)$ for $P$ is strong if each point in $P$ sees only differently colored guards. $(G, \gamma)$ is a conflict-free guarding if for any point $p \in P$ there is at least one guard in the guard set $G(p)=V(p) \cap G$ whose color is unique among all guards visible from $p$.

Figure 1 illustrates both concepts. We denote by $\chi_{c f}(P)$ the minimal $t$ such that there is a conflict-free chromatic guarding set for $P$ using $t$ colors. Maximizing this value over all polygons with $n$ vertices from a specified polygon class is denoted by $\chi_{c f}(n)$.

Consequently, we denote by $\chi_{s t}(P)$ the minimal $t$ such that there is strong chromatic guarding set using $t$ colors. Maximizing this value for all polygons with $n$ vertices from a specified polygon class defines the value $\chi_{s t}(n)$. Observe that minimizing the guard number is not part of the objective function. However, in our upper bound proofs we use at most a linear number of guards, which is asymptotically optimal in worst case.

\section{Upper Bounds}

We show two upper bounds for simple orthogonal polygons of size $n$ in the r-visibility model: $\chi_{s t}^{r}(n) \in O(\log n)$ and $\chi_{c f}^{r}(n) \in O(\log \log n)$. These bounds are even realized by guards placed in the interior of visibility cells. This restriction simplifies the arguments and does not affect the asymptotic bounds. Furthermore we use the simple fact that a polygon is guarded iff its interior is guarded. The upper bound proof is inspired by ideas developed in [1,2] for conflict-free guarding of simple polygons based on line visibility.

\subsection{Reduction to histograms}

We reuse the central concept of independence introduced in $[1,2]$ for line visibility. Independence means that one can use the same color sets for coloring guards in independent subpolygons. The following definition suffices for our purposes and covers both the strong and the conflict-free variant:

Let $P$ be a simple orthogonal polygon and $P_{1}$ and $P_{2}$ subpolygons of $P$. We call $P_{1}$ and $P_{2}$ independent if no point in $P$ can simultaneously see points from $\operatorname{int} P_{1}$ and $\operatorname{int} P_{2}$.

Next, we hierarchically subdivide an orthogonal polygon $P$ into a linear number of histograms by a standard window partitioning process, see [1]. For the sake of simplicity we make the (weak) assumption that the obtained histograms have no degenerate edges.

The subdivision is represented by a partition tree $\mathcal{H}=\mathcal{H}_{P}(e)$ with histograms as node set. Let $e$ be a highest horizontal boundary edge. The visibility polygon of $e$ is a histogram $Q$. This is the root vertex of $\mathcal{H}$. Now $Q$ splits $P$ into parts and defines a finite set (possibly empty if $Q=P$ ) of vertical windows $w_{1}, \ldots, w_{k}$. Then we recurse, see Figure 2 , with the windows being the new base edges. Each window corresponds to a last left or right turn of a shortest orthogonal path from $e$ to the histogram defined by the window. So we can accordingly label the histograms to be left or right histograms. We define the root $Q$ to be a left histogram.

Let $H_{d}, d=0,1,2$, be the family of all histograms corresponding to nodes in $\mathcal{H}$ with depth congruent $d \bmod 3$. We further partition $H_{d}$ into $H_{d}^{L}$ and $H_{d}^{R}$ depending on whether the histograms are left or right histograms, respectively. In Figure 2 the six families of histograms are color-coded for illustration. For example, the dark gray histograms are right children with depth congruent $1 \bmod 3$. 

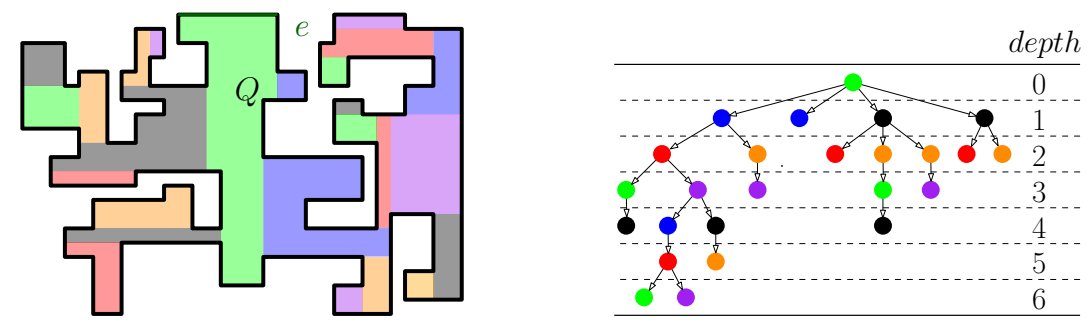

Figure 2 The partition into histograms and the corresponding partition tree.
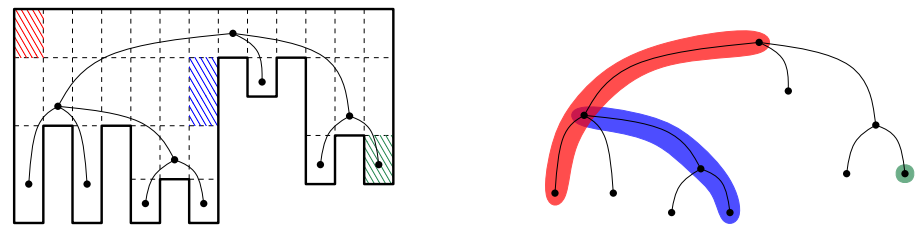

Figure 3 Spine tree and the bijection between open cells and monotone paths.

- Lemma 1. Let $P$ be a polygon and $H_{d}^{L}, d=0,1,2$ the family of histograms corresponding to left nodes in $\mathcal{H}$ with depth congruent $d \bmod 3$. Then the interior of histograms in each $H_{d}^{L}$ have pairwise orthogonal link distance at least three, analogously for $H_{d}^{R}$, so they are independent.

\subsection{Guarding a histogram}

Consider a histogram $H \subseteq P$ with a top horizontal base edge. We associate with $H$ a tree $T$ as follows. Consider the set of cells in the r-visibility arrangement $\mathcal{A}^{r}$. If several cells have the same visibility polygon we choose the leftmost cell as representative of this equivalence class. Let $R$ be the set of all representatives and $B \subseteq R$ the subset of cells incident to bottom horizontal histogram edges. We define a partial order for $B$ : We say $b^{\prime} \leq b$ iff the horizontal polygon edge of $b^{\prime}$ is not above that of $b$ and there is an $r \in R$ that sees both $b$ and $b^{\prime}$. The Hasse diagram of this poset is a tree $T$ which we call the spine tree of $H$. A monotone path $\pi$ in $T$ is a directed subpath of a root-to-leaf path. It corresponds to a pair $\left(b, b^{\prime}\right)$ with $b^{\prime} \leq b$.

- Lemma 2. There is a bijective mapping $\Phi$ between cells of $R$ and monotone paths in $T$ such that two cells are visible from each other iff the corresponding monotone paths in $T$ share a node.

Proof. Let $r$ be a cell in $R$. Then $V(r) \cap T$ is some monotone path $\pi$ in $T$ and we set $\Phi(r)=\pi$. For the inverse function let $\pi$ be a monotone path in $T$ from vertex $b$ down to $b^{\prime}$. We associate with $\pi$ the unique cell $\Phi^{-1}(\pi)=r \in R$ that is vertically aligned with $b^{\prime}$ and horizontally with $b$.

We observe that $\Phi$ is well-defined by the choice of the leftmost representative for visibility equivalent cells and it is clearly a bijection. Especially, for $\pi=(b, b)$ we have $\Phi^{-1}(\pi)=b$.

For the second assertion consider two cells $r, r^{\prime}$ visible from each other and the smallest rectangle that includes both. By extending this rectangle downwards it hits a horizontal boundary edge. The vertex of $T$ corresponding to that edge is in both $\Phi(r)$ and $\Phi\left(r^{\prime}\right)$. For the other direction consider a cell $r^{\prime \prime}$ corresponding to a vertex in $\Phi(r) \cap \Phi\left(r^{\prime}\right)$. It has a bottom horizontal edge. We form a rectangle in $H$ above this edge of maximal width and maximal height. All cells visible from $r^{\prime \prime}$ are in this rectangle, therefore $r$ sees $r^{\prime}$. Figure 3 illustrates the bijection. 


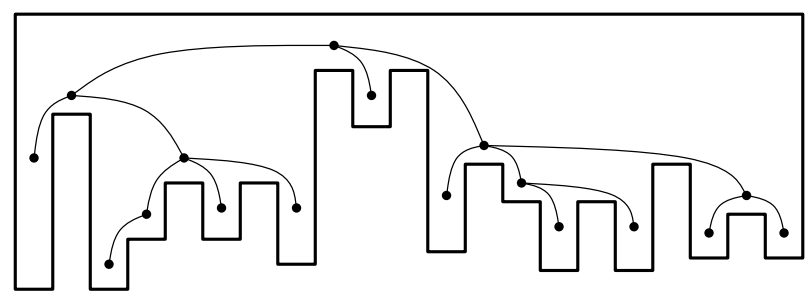

Figure 4 Example histogram with spine tree.

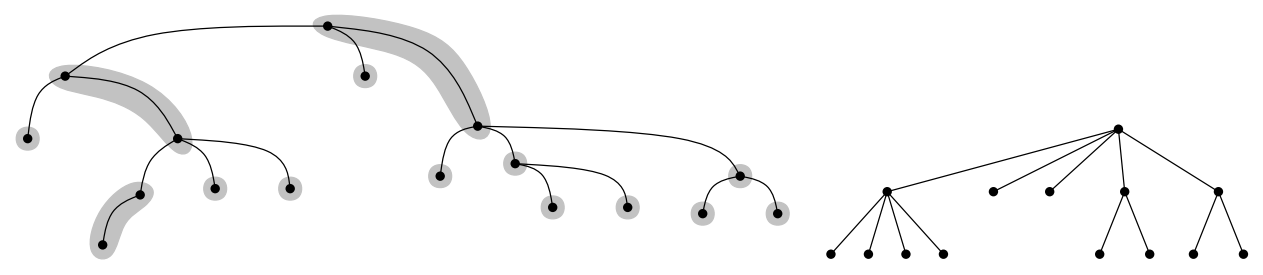

Figure 5 Monotone paths covering the spine tree and the corresponding compressed spine tree.

Now we translate the geometric concepts of strong and conflict-free guardings of $H$ into equivalent combinatorial questions for the spine tree $T$. First of all, a colored guard set for $H$ defines a set of colored cells in $R$ and this defines, using $\Phi$, a covering of $T$ with colored monotone guarding paths and vice versa. The condition for strong guarding now reads: No monotone path in $T$ can intersect two guarding paths of the same color.

For conflict-free guardings we have:

- Lemma 3. Colored guards $g_{1}, \ldots, g_{r}$ define a conflict-free guarding for $H$ iff for each monotone path $\pi$ in $T$ there exists a color and exactly one guarding path $\Phi\left(g_{i}\right)$ with that color such that $\pi \cap \Phi\left(g_{i}\right) \neq \emptyset$.

Proof. Consider the cell $\Phi^{-1}(\pi)$. It is seen by a guard $g$ with a unique color $c$. Therefore $\Phi(g) \cap \pi \neq \emptyset$. Assume, some other $c$-colored guarding path $\Phi\left(g^{\prime}\right)$ intersects $\pi$. Then $g^{\prime}$ sees $\Phi^{-1}(\pi)$, a contradiction. The other direction is analogous.

Path compression: We use a bottom-up path compression to define a covering (in fact, it is a partition) of $T$ by monotone paths. To this end we form, in parallel, for all leaves $l$ the maximal length monotone paths $\pi(l)$ that end in $l$ and do not use nodes of outdegree bigger than one. We cut off all $\pi(l)$ from $T$. Iterating this procedure yields a unique tree $T^{*}$. Its nodes represent monotone paths in $T$. $T^{*}$ has depth $O(\log |H|)$ since in each iteration the number of leaves is reduced by at least half. Figure 4 shows an example histogram with its spine tree $T$. The derived compressed spine tree is depicted in Figure 5.

The above path compression technique is similar but not equivalent to that of heavy path decompositions [13]. In fact, the same bounds can be achieved using the (heavy) path tree of heavy path decompositions as $T^{*}$.

- Proposition 4. Let $H$ be a histogram with $n$ vertices. In the r-visibility model there is a strong chromatic guarding with $O(\log n)$ colors and a conflict-free chromatic guarding with $O(\log \log n)$ colors.

Proof. We construct the spine tree $T$ and the compressed tree $T^{*}$ with depth $O(\log n)$. To get a strong guarding we color the nodes of $T^{*}$, i.e. the guarding paths in $T$, by their depth in $T^{*}$. 


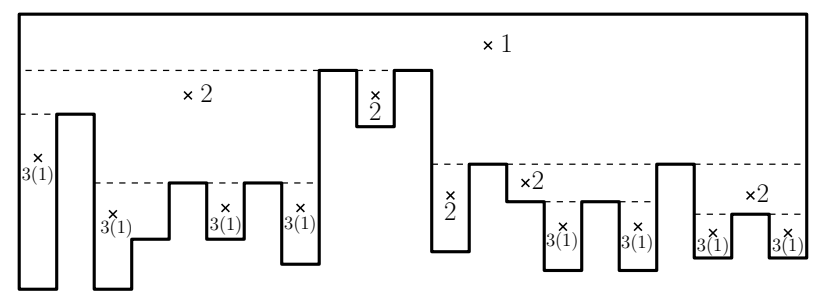

Figure 6 Chromatic guarding positions for the example histogram: with colors $\{1,2,3\}$ strong, with colors $\{1,2\}$ conflict-free (in brackets) guarding.

For a conflict-free guarding, consider the color set $[t]=\{1,2, \ldots, t\}$ and the following recursively defined set of words: $s_{1}=1$ and $s_{i}=s_{i-1} \circ i \circ s_{i-1}$. Clearly, a prefix of $s_{t}$ with length $k$ has no more than $\lceil\log (k+1)\rceil$ different colors and each connected subword contains a unique color [14]. Now we color the nodes of $T^{*}$ from the root to the leaves with the sequence $s_{t}$ of length at most the height of the tree, that is $O(\log n)$. A color alphabet of size $O(\log \log n)$ suffices.

We illustrate the construction in Figure 6. Observe that we use the same guard positions for both strong and conflict-free guarding. The compressed spine tree has depth 2 . For the strong guarding we use colors 1,2 and 3 while for the conflict-free version we use the color sequence 1-2-1. The guard positions are in the open cells corresponding to the monotone paths via bijection $\Phi$. Moreover, each guard covers a pyramid as indicated in the figure.

- Theorem 5. Let $P$ be an orthogonal polygon with $|P|=n$. We have $\chi_{s t}^{r}(n) \in O(\log n)$ and $\chi_{c f}^{r}(n) \in O(\log \log n)$.

Proof. We decompose $P$ into 6 families $H_{d}^{L}, H_{d}^{R}, d=0,1,2$. Each of the families consists of pairwise independent histograms each of size at most $n$. Then we apply Proposition 4 .

\section{$4 \quad$ Lower Bounds}

This section contains three lower bound proofs, two tight lower bounds for strong and for conflict-free r-visibility guards, and a first non-trivial lower bound for conflict-free l-visibility guards in simple polygons. All use the same underlying orthogonal histogram but they completely differ with respect to proof techniques. Both r-visibility cases rely on the spine tree concept from Section 3.2. For line visibility guards we introduce a new combinatorial method which we call multicolor tableau.

\subsection{Lower bounds for r-visibility}

All lower bounds established in this paper are based on a simple, recursively defined family of so called spike polygons $S_{m}$, where $S_{1}$ is a simple square and $S_{m+1}$ is formed by two copies of $S_{m}$ separated by a vertical spike, but joined by an additional horizontal layer. Figure 7 illustrates this construction together with the subdivision of $S_{2}$ into visibility cells. Obviously, the spine tree $T$ of $S_{m}$ is a balanced binary tree of height $m-1$ with vertices corresponding one-to-one to bottom cells in the r-visibility arrangement. Recall, a colored r-visibility guard set for $S_{m}$ corresponds to a covering of $T$ with colored monotone guarding paths and vice versa.

- Theorem 6. For simple orthogonal polygons $\chi_{s t}^{r}(n) \in \Omega(\log n)$. 
Proof. We show that any strong guarding of $S_{m}$ requires $m$ different colors. Consider in the spine tree $T$ a guarding path $\pi$ covering the root with unique color $c$. Then $c$ does not occur in the left or in the right subtree of the root. By induction we need $m-1$ more colors for the subtree missed by $\pi$. Since $S_{m}$ has $n=2^{m+1}$ vertices, the claim follows.

Next we consider a lower bound for the conflict-free version of the problem. To that end, we analyze the special case that a root-to-leaf path $\pi$ in $T$ is covered by short guarding paths only. Later we show the existence of such a path.

- Lemma 7. Let $\mathcal{P}=\left\{\pi_{1}, \ldots, \pi_{r}\right\}$ be conflict-free guarding paths for a path $\pi$ with $m$ nodes such that $\left|\pi_{i}\right|=O\left(m^{\epsilon}\right)$ for $1 \leq i \leq r$ and some $0<\epsilon<1$. Then this guarding uses at least $\Omega(\log m)$ colors.

Proof. Let $K=\max \left\{\left|\pi_{i}\right|, 1 \leq i \leq r\right\}$. We subdivide $\pi$ into $k=\frac{m}{K} \in \Omega\left(m^{1-\epsilon}\right)$ buckets of size $K$ each. This way every $\pi_{i}$ can overlap with at most two buckets. Since $\mathcal{P}$ is induced by a conflict-free guarding, there is a color $c_{1}$ such that exactly one $\pi_{i}$ (responsible for $\pi$ ) is colored with $c_{1}$. Hence there is a subpath of $\pi$ consisting of at least $\frac{k-2}{2}$ buckets that does not intersect any $c_{1}$-colored path. Applying this argument recursively we obtain the following recursive relation for the number of colors needed for $k$ buckets: $T(k) \geq T\left(\frac{k-2}{2}\right)+1$. This recursive relation easily solves to $T(k) \in \Omega(\log k) \subseteq \Omega\left(\log m^{1-\epsilon}\right)=\Omega(\log m)$.

- Theorem 8. For simple orthogonal polygons $\chi_{c f}^{r}(n) \in \Omega(\log \log n)$.

Proof. We start with a conflict-free guarding of $S_{m}, n=2^{m+1}$ that uses a minimum number of $t$ colors. By Theorem 5 we have $t \in O(\log \log n)=O(\log m)$. Consider the corresponding family $\mathcal{F}$ of guarding paths in $T$. We denote by $\mathcal{U}\left(v_{0}\right)$ the set of all guarding paths from $\mathcal{F}$ covering the root $v_{0}$ of $T$ with a unique color. Since $\left|\mathcal{U}\left(v_{0}\right)\right| \leq t$ there must be a vertex $v_{1}$ at depth $\lfloor\log t\rfloor+1$ that is not part of any path from $\mathcal{U}\left(v_{0}\right)$. Now we iterate starting from $v_{1}$. We take all guarding paths covering $v_{1}$ with a unique color and we determine a node $v_{2}$ at depth $2\lfloor\log t\rfloor+2$ missed by these paths, and so on. We call the $v_{i}$ 's checkpoints. The checkpoints define a root-to-leaf path $\pi$ with length $m=\log n-1$. Consider $\mathcal{F}_{\pi}=\left\{\pi \cap \pi_{i} \mid \pi_{i} \in \mathcal{F}\right\}$. Now form a new family $\mathcal{U}_{\pi}$ that consists of all maximal subpaths $\sigma$ of members $\pi_{i} \cap \pi \in \mathcal{F}_{\pi}$ such that $\sigma$ does not intersect any other member of the same color in $\mathcal{F}_{\pi}$. Let $\pi^{\prime} \subset \pi$ and assume $\pi_{i} \in \mathcal{F}$ gives a unique color to $\pi^{\prime}$. Then $\pi^{\prime} \cap \pi_{i}$ is part of some path in $\mathcal{U}_{\pi}$. Thus $\mathcal{U}_{\pi}$ is a conflict-free guarding path family for $\pi$. By construction, paths in $\mathcal{U}_{\pi}$ have length at most $2\lfloor\log t\rfloor+1$. Now we can apply Lemma 7 . Since $2 \log t+1 \in O(\log \log m) \subseteq O\left(m^{0.5}\right)$ we get $t \in \Omega(\log m)=\Omega(\log \log n)$.

\subsection{Blocks, stretched spike polygons, and multicolor tableaux}

We now turn our attention to conflict-free chromatic guarding in the line visibility model. The concepts needed in our lower bound proof are explained in this section.

Columns of $S_{m}$ are numbered left to right by indices $k \in\left[2^{m}-1\right]$, and cells in column $k$ top down by an additional index $i \in\left[d_{m}(k)\right]$ where $d_{m}(k)$ is the depth of column $k$ in $S_{m}$. Formally, we have $d_{m}(k)=m-\pi_{2}(k)$, where $\pi_{2}(k)$ is the multiplicity of factor 2 in the prime decomposition of $k$. Obviously, a column has maximal depth $m$ iff its index is odd. We introduce the notions of the left and right wing of column $k$ in order to distinguish guard positions: The left wing $W_{L}(k)$ is the set of all points strictly on the left side of the midline of column $k$ and the right wing is the complement $W_{R}(k)=S_{m} \backslash W_{L}(k)$ including the midline. 

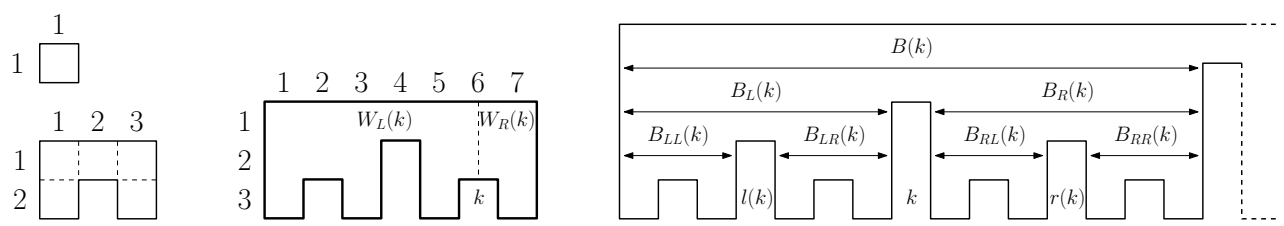

Figure 7 Spike polygons $S_{1}$ and $S_{2}$ (left), left wing and right wing of column $k=6$ in $S_{3}$ (middle), blocks and subblocks (right).

We define the block $B(k)$ of column $k$ as the interval of all neighboring columns of depth at least $d_{m}(k)$, see Figure 7:

$$
B(k)=\left[k-\left(2^{\pi_{2}(k)}-1\right), k+\left(2^{\pi_{2}(k)}-1\right)\right]
$$

Geometrically, a block is nothing but a smaller spike polygon. Deleting its central column a block splits into a left and a right subblock:

$$
B_{L}(k)=\left[k-\left(2^{\pi_{2}(k)}-1\right), k-1\right] \quad, \quad B_{R}(k)=\left[k+1, k+\left(2^{\pi_{2}(k)}-1\right)\right]
$$

For odd $k$ we have $B(k)=\{k\}$ and $B_{L}(k)=B_{R}(k)=\emptyset$. Later it will be necessary to subdivide a left or right subblock again into its left and right subblocks. These quartersubblocks can be described making use of the definition above together with the central column $l(k)=k-2^{\pi_{2}(k)-1}$ in block $B_{L}(k)$ and column $r(k)=k+2^{\pi_{2}(k)-1}$ in block $B_{R}(k)$ :

$$
B_{L L}(k)=B_{L}(l(k)), B_{L R}(k)=B_{R}(l(k)), B_{R L}(k)=B_{L}(r(k)), \quad B_{R R}(k)=B_{R}(r(k)) .
$$

Next we introduce a vertically stretched, but combinatorially equivalent version $S_{m}^{\downarrow}$ of $S_{m}$ with the following properties:

1. The width of each column is 1 and hence the total width of $S_{m}^{\downarrow}$ is again $2^{m}-1$.

2. We will distinguish between combinatorial and geometric depth of a column: While $d_{m}(k)=m-\pi_{2}(k)$ is still the combinatorial depth, we want the geometric depth to be $d_{m}^{\downarrow}(k)=2^{\left(d_{m}(k)-1\right) m}$. That means that the height of the first row is $h_{1}=1$ and the height of the $i$-th row $h_{i}=2^{i m}-2^{(i-1) m}$.

Consider the r-visibility(!) arrangement of $S_{m}^{\downarrow}$ with the rectangular r-visibility cells $r_{i, k}$. Next we discretize the conflict-free l-guarding problem. Let $p_{i, k}$ be the bottom side midpoint of cell $r_{i, k}$, that is the cell in row $i$ and column $k$. If $\gamma: G \rightarrow[t]$ for a guard set $G$ is a conflict-free l-guarding of $S_{m}^{\downarrow}$, then let $M_{i, k}$ be the multiset of all colors of guards that see point $p_{i, k}$. By $m_{i, k}(c)$ we denote the multiplicity of color $c$ in this multiset.

We call $\mathcal{M}(\gamma)=\left\{M_{i, k} \mid k \in\left[2^{m}-1\right], i \in\left[d_{m}(k)\right]\right\}$ the corresponding multicolor tableau. The set of unique guard colors for point $p_{i, k}$ is defined by $U_{i, k}:=\left\{c \in[t] \mid m_{i, k}(c)=1\right\}$ and the standard inclusion relation $M_{i, k} \subseteq M_{j, l}$ for multisets by: $\forall c \in[t] \quad m_{i, k}(c) \leq m_{j, l}(c)$. The next two lemmata state simple l-visibility properties in stretched spike polygons.

- Lemma 9. Let $g$ be an l-guard in $S_{m}^{\downarrow}$ and $k$ a column of this polygon with combinatorial depth $d=d_{m}(k)$ and geometric depth $d_{m}^{\downarrow}(k)=2^{(d-1) m}$. If $g \in W_{R}(k)\left(g \in W_{L}(k)\right)$ then $g$ cannot see any point $p$ at depth $d^{\downarrow}(p) \geq 2^{d m}$ in the left (right) block of $k$. In particular, $g$ cannot see any point $p_{i, j}$ with $j \in B_{L}(k)\left(j \in B_{R}(k)\right)$ and $i>d$.

Proof. By symmetry it is sufficient to study the first case with $g \in W_{R}(k), d^{\downarrow}(p) \geq 2^{d m}$ and $p$ a point in the subpolygon $B_{L}(k)$. Let $q_{L}$ be the left vertex of the horizontal polygon edge 

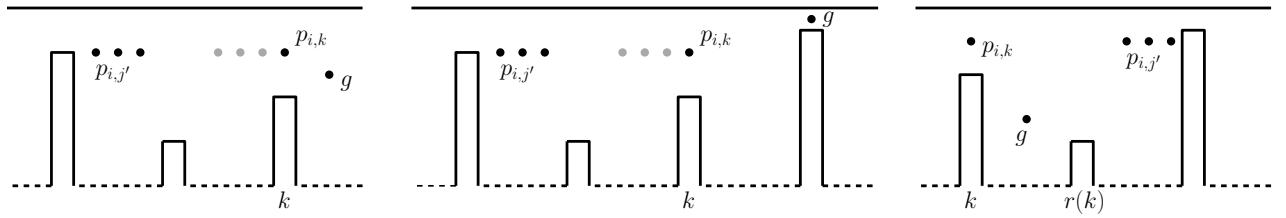

Figure 8 Possible guard positions with respect to the point $p_{i, k}$. Note that it is impossible to display the exponential growth of the row heights in the drawing.

in column $k$ and consider the slopes $s_{1}$ and $s_{2}$ of the lines $\overline{p q_{L}}$ and $\overline{q_{L} g}$. Since the width of $B_{L}(k)$ is $2^{m-d}-1$ and $d^{\downarrow}(p)-d^{\downarrow}\left(q_{L}\right) \geq 2^{d m}-2^{(d-1) m}=\left(2^{m}-1\right) \cdot 2^{(d-1) m}$ we get

$$
s_{1} \geq \frac{\left(2^{m}-1\right) \cdot 2^{(d-1) m}}{2^{m-d}-1}=\frac{\left(2^{m}-1\right) \cdot 2^{(d-1) m}}{2^{-d}\left(2^{m}-2^{d}\right)}>\frac{2^{(d-1) m}}{2^{-d}}=2^{(d-1) m+d}
$$

Since $g$ is in the right wing of $k$ it is at least one half unit right of $q_{L}$ and it is at most $d_{m}^{\downarrow}(k)=2^{(d-1) m}$ units higher than $q_{L}$. We get

$$
s_{2} \leq \frac{2^{(d-1) m}}{1 / 2}=2^{(d-1) m+1} \leq 2^{(d-1) m+d}
$$

Thus $s_{1}>s_{2}$, which shows that vertex $q_{l}$ blocks the l-visibility between $p_{i, j}$ and $g$.

- Lemma 10. Let $g$ be an l-guard watching a point $p_{i, k} \in S_{m}^{\downarrow}$. Then, for all $i^{\prime} \leq i$ and for all $j \in B_{L}(k)$ or for all $j \in B_{R}(k)$, g sees also $p_{i^{\prime}, j}$.

Proof. Let $d^{\downarrow}(g)$ be the geometric depth of $g$ in $S_{m}^{\downarrow}$.

Case 1: (Fig. 8, left) If $g$ is even an r-guard for $p_{i, k}$ then the rectangle spanned by $g$ and $p_{i, k}$ can be horizontally extended over the whole block $B(k)$ as well as upwards to the top of $S_{m}^{\downarrow}$. Thus the claim holds for all $j \in B(k)$.

Otherwise there are two more cases, namely that $d^{\downarrow}(g)$ is strictly smaller or strictly larger than $2^{(i-1) m}$.

Case 2: (Fig. 8 , middle) $d^{\downarrow}(g)<2^{(i-1) m}$, i.e., $g$ sees $p_{i, k}$ from above. If $g \in W_{R}(k)$ then $g$ can see all $p_{i, j}$ with $j \in B_{L}(k)$ because the line segments $p_{i, j} p_{i, k}$ and $p_{i, k} g$ are contained in $S_{m}^{\downarrow}$ and they form a chain that is convex from above. If $g \in W_{L}(k)$ then $g$ can see all $p_{i, j}$ with $j \in B_{R}(k)$ beause the line segments $g p_{i, k}$ and $p_{i, k} p_{i j}$ are contained in $S_{m}^{\downarrow}$ and they form a chain that is convex from above. Moreover it is clear that in $S_{m}^{\downarrow}$ any guard that sees a point $p_{i, j}$ will see also all points directly above, especially the points $p_{i^{\prime}, j}$ with $i^{\prime}<i$.

Case 3: (Fig. 8, right) $d^{\downarrow}(g)>2^{(i-1) m}$, i.e., $g$ sees $p_{i, k}$ from below. We can additionally assume $d^{\downarrow}(g)>d_{m}^{\downarrow}(k)=2^{\left(d_{m}(k)-1\right) m}$ since otherwise we are in Case 1 again. By Lemma 9 (with the roles of guard and guarded point exchanged) we know that $g$ is in row $i^{\prime \prime}=d_{m}(k)+1$ in some cell $r_{i^{\prime \prime}, j}$ with $j \in B_{L}(k)$ or $j \in B_{R}(k)$. It follows that, depending on whether $g$ lies in $B_{L}(k)$ or $B_{R}(k), g$ sees all $p_{i^{\prime \prime}, j^{\prime}}$ with $j^{\prime} \in B_{L}(k)$ or $j^{\prime} \in B_{R}(k)$ and all points above.

A multicolor tableau $\mathcal{M}(\gamma)$ has standard size if it consists of $m$ rows and $N=2^{m}-1$ columns. But by various constructions, for example restricting it to a single block, one creates a new tableau having $m$ rows and $N^{\prime}=2^{m^{\prime}}-1$ columns for some $m^{\prime}<m$.

The following central definition of $t$-conformity specifies some necessary, but not sufficient conditions a multicolor tableau has if it stems from a conflict-free $t$-chromatic l-guarding of a stretched spike polygon. Later we will show that $t$-conformity is preserved when acting on the tableau with various combinatorial operations defined below. 
- Definition 11. Let $m^{\prime} \leq m$ be natural numbers and $N^{\prime}=2^{m^{\prime}}-1$. A combinatorial scheme of multisets over the ground set $[t]$ of the form $\mathcal{M}=\left(M_{i, k} \mid k \in\left[N^{\prime}\right], i \in\left[d_{m}(k)\right]\right)$ is called a $t$-conform $m \times N^{\prime}$ multicolor tableaux if the following properties hold:

1. cf-Property: $\forall_{k \in\left[N^{\prime}\right]} \forall_{i \in\left[d_{m}(k)\right]} U_{i, k} \neq \emptyset$.

2. Monotonicity: $\forall_{k \in\left[N^{\prime}\right]} \forall_{1 \leq i<i^{\prime} \leq d_{m}(k)} M_{i^{\prime}, k} \subseteq M_{i, k}$.

3. LR-quarter-block property: If $c$ is a unique color for some point $p_{i, k}$ in column $k$ then there exists a quarter-subblock $B_{X Y}(k)$ with $X Y \in\{L L, L R, R L, R R\}$ such that for all columns $j \in B_{X Y}(k)$ the following predicate $Q(j)$ holds. $Q(j)$ is the conjunction of three conditions:
a. $c \in M_{i, j}$
b. $c \in U_{i, j} \rightarrow c \notin M_{d_{m}(k)+2, j}$
c. $c \notin U_{i, j} \rightarrow \exists_{Z \in\{L, R\}} \forall_{j^{\prime} \in B_{Z}(j)} c \notin U_{i, j^{\prime}}$.

- Proposition 12. The multicolor tableau $\mathcal{M}(\gamma)$ for a conflict-free l-guarding $\gamma$ of the polygon $S_{m}^{\downarrow}$ with $t$ colors is $t$-conform.

Proof. There is nothing to prove for the cf-property and the monotonicity. Now let us assume $c \in U_{i, k}$ with a corresponding guard $g$. By symmetry we may suppose $g \in W_{R}(k)$. Again, there are three cases to distinguish (see Figure 8):

1. $p_{i, k}$ is r-visible from $g$.

2. $p_{i, k}$ is not r-visible from $g$ and $p_{i, k}$ is deeper than $g$.

3. $p_{i, k}$ is not r-visible from $g$ and $g$ is deeper than $p_{i, k}$.

In Case 1 and Case 2 we choose $X Y=L L$ (but $X Y=L R$ would also work - the gray points). In Case 3 the choice depends on the position of $g$ relative to the central column $r(k)$ of the block $B_{R}(k)$ :

$$
X Y= \begin{cases}R L & \text { if } g \in W_{R}(r(k)) \\ R R & \text { if } g \in W_{L}(r(k)) .\end{cases}
$$

It remains to establish the three conditions of $Q(j)$ for all $j \in B_{X Y}(k)$. Condition (a) is obvious in Case 1 and Case 2. In Case 3 it follows from the fact that $g$ cannot be deeper than $d_{m}^{\downarrow}(r(k))$, see the proof of Lemma 9 .

For condition (b) suppose that $c \in U_{i, j}$. This implies that $g$ is the only guard with color $c$ that sees $p_{i, j}$. However, in all three cases $g$ is in the wing opposite to block $B_{X Y}(k)$ and then $g$ cannot see any point of combinatorial depth $d_{m}(k)+2$ in $B_{X Y}(k)$ by Lemma 9 . It is worth observing that depth $d_{m}(k)+1=d_{m}(r(k))$ does not suffice in Case 3. Any other $c$-colored guard watching $p_{d_{m}(k)+2, j}$ would also watch $p_{i, j}$ and therefore contradicts the uniqueness of $g$. Thus $c \notin M_{d_{m}(k)+2, j}$.

Finally, let us suppose $c \notin U_{i, j}$, then there is a second $c$-colored guard $g^{\prime}$ for $p_{i, j}$. Now we can conclude from Lemma 10 that $g^{\prime}$ watches all points $p_{i, j^{\prime}}$ for $j^{\prime} \in B_{L}(j)$ or for all $j^{\prime} \in B_{R}(j)$. This proves condition (c).

\subsection{The lower bound proof for I-visibility}

We start with describing operations on $t$-conform multicolor tableaux that maintain this property. In Figure 9 the three operations are geometrically illustrated, however, the operations themselves are defined for the combinatorial tableaux.

- Proposition 13. If $\mathcal{M}=\left(M_{i, k} \mid k \in\left[N^{\prime}\right], i \in\left[d_{m}(k)\right]\right)$ is a t-conform $m \times N^{\prime}$ tableau with $N^{\prime}=2^{m^{\prime}}-1$ for some $m^{\prime} \leq m$, then the following three constructions yield new $t$-conform tableaux $\mathcal{M}_{1}, \mathcal{M}_{2}, \mathcal{M}_{3}$ : 

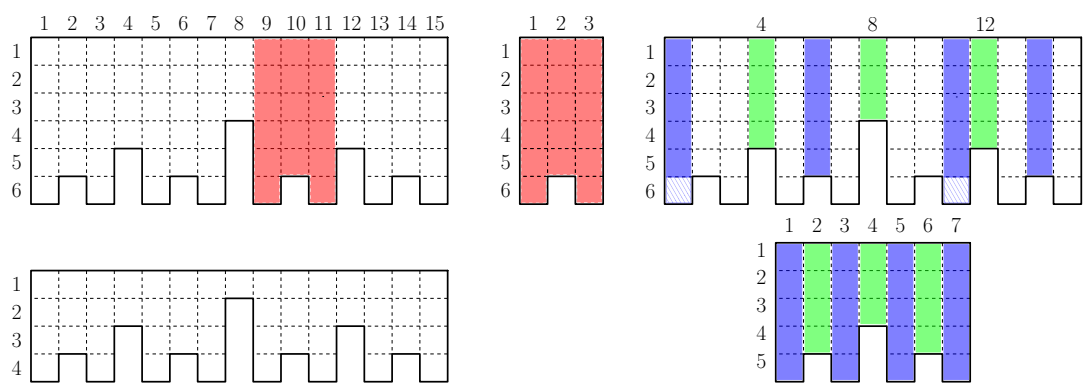

Figure 9 Illustrating horizonal (red), vertical (lower left), and selective (blue-green) truncation of a $6 \times 15$ multicolor tableau.

1. Horizontal truncation: $\mathcal{M}_{1}$ results from restricting $\mathcal{M}$ to a block $B(k)$;

2. Vertical truncation: $\mathcal{M}_{2}$ results from deleting the top $m-m^{\prime}$ rows of $\mathcal{M}$;

3. Selective truncation: $\mathcal{M}_{3}$ results from selecting $2^{m^{*}}-1$ columns for some $m^{*}<m^{\prime}$ with respect to the following rules:

- For all even $k \in\left[2^{m^{*}}-1\right]$ choose column $k \cdot 2^{m^{\prime}-m^{*}}$ of $\mathcal{M}$ as column $k$ of $\mathcal{M}_{3}$.

- For all odd $k \in\left[2^{m^{*}}-1\right]$ choose any column $j$ of $\mathcal{M}$ with $(k-1) \cdot 2^{m^{\prime}-m^{*}}<j<$ $(k+1) \cdot 2^{m^{\prime}-m^{*}}$, delete from that column all entries of depth $d>m^{*}+m-m^{\prime}$ and use this truncated column as column $k$ of $\mathcal{M}_{3}$.

Proof. Recall, the width of $B(k)$ is $N^{\prime \prime}=2^{m^{\prime \prime}}-1$ where $m^{\prime \prime}=2 \pi_{2}(k)$. So the only thing that one has to do for $\mathcal{M}_{1}$ is shifting the column indexing from the interval $B(k)=$ $\left[k-2^{\pi_{2}(k)}+1, k+2^{\pi_{2}(k)}-1\right]$ to $\left[N^{\prime \prime}\right]$. Then $\mathcal{M}_{1}$ is t-conform.

For the second construction it is sufficient to shift down by $m-m^{\prime}$ the index of each row that is not deleted. Then $\mathcal{M}_{2}$ is an $m^{\prime} \times N^{\prime}$ tableau. Note that an old row index $d_{m}(k)=m-\pi_{2}(k)$ becomes $d_{m^{\prime}}(k)$. Having that in mind, it is also trivial that $\mathcal{M}_{2}$ is t-conform.

The construction of $\mathcal{M}_{3}$ already contains the renumbering of indices. Again, it is not hard to conclude the $t$-conformity because the construction preserves the relation of being a column in the left (or right) subblock of another column.

- Theorem 14. $\chi_{c f}^{l}(n) \in \Omega\left(\frac{\log \log n}{\log \log \log n}\right)$.

Proof. We define a recursive function $m(t)$ by $m(1)=3$ and $m(t)=1+t \cdot(m(t-1)+1)$ for $t \geq 2$.

The inequality $m(t) \leq(t+1)$ ! holds for all $t \geq 5$ by induction. In fact, $m(5)=651<720=$ $(5+1)$ ! and the induction step works for any $t \geq 6$ as follows:

$$
m(t)=t \cdot(m(t-1)+1)+1 \leq t(t !+1)+1=t \cdot t !+(t+1) \leq t \cdot t !+t !=(t+1) !
$$

Claim: An $m(t) \times\left(2^{m(t)}-1\right)$ tableau cannot be $t$-conform.

Before proving this claim we first show how it implies the Theorem. By Proposition 12 and the Claim we have $\chi_{c f}^{l}(n)>t$ for all $t \geq 5$ and some $n \leq 2^{(t+1) !+1}$, since $2^{(t+1) !+1}$ is an upper bound on the number of vertices in $S_{m(t)}^{\downarrow}$. This implies $\log \log n \in O(t \log t)$ and finally $t \in \Omega\left(\frac{\log \log n}{\log \log \log n}\right)$.

The proof of the Claim is by induction on $t$. The induction base is for $t=1$. We show it by contradiction. Any 1-conform $3 \times 7$ tableau requires to set $U_{i, k}=\{1\}$ for all $k \in[7]$ and 
all $i \in\left[d_{3}(k)\right]$. However, looking at the LR-quarter-block property for the situation $1 \in U_{1,4}$ yields a contradiction with condition (b).

The induction step is also proved by contradiction. Assume that there are no $(t-1)$ conform $m^{\prime} \times N^{\prime}$ tableaux with $m^{\prime}=m(t-1)$ and $N^{\prime}=2^{m^{\prime}}-1$, but there is a $t$-conform $m \times N$ tableau $\mathcal{M}$ for $m=m(t)$ and $N=2^{m}-1$.

The following reasoning is a bit involved, so we first give an overview.

Outline: The proof by contradiction consists of $s$ stages, for some $1 \leq s \leq t$. The precondition of stage $s$ is the existence of a $t$-conform $m \times N_{s-1}$ tableau where $N_{s-1}=$ $2^{m-(s-1)\left(m^{\prime}+1\right)}-1$ such that the following additional property holds. There is a color set $C_{s-1} \subseteq[t]$ consisting of $s-1$ colors, such that for all these $c \in C_{s-1}$ and for all columns $k \in\left[N_{s}\right]$ holds $c \notin U_{1, k}$. The precondition for the first stage is given by the tableau $\mathcal{M}$ with $N_{0}=N$ and $C_{0}=\emptyset$. The tableau $\mathcal{M}$ will change after every stage. The postcondition of the $s$-th stage is either a contradiction obtained by constructing a $(t-1)$-conform $m^{\prime} \times N^{\prime}$ tableau (this is Case 1: the stop condition) or the validation of the precondition for the next stage (this is Case 2). This will work in such a way that if the stop condition does not occur even after the $t$-th stage, then the derived condition also yields a contradiction. We would then have $C_{t}=[t]$ and $N_{t}=2^{1}-1=1$, i.e., it results in a $t$-conform $m \times 1$ tableau (i.e., a single column) such that no color can be unique in $M_{1,1}$.

Proof details: Suppose that an $m \times N_{s-1}$ tableau $\mathcal{M}$ with a color set $C_{s-1}$ fulfills the precondition for stage $s$ with $1 \leq s \leq t$. Let $k=\frac{N_{s-1}+1}{2}$ be the central column of $\mathcal{M}$ and $c_{s} \in U_{1, k}$. Note that the precondition implies $c_{s} \notin C_{s-1}$. By the LR-quarter-block property of $t$-conform tableaux there is some $X Y \in\{L L, L R, R L, R R\}$ such that predicate $Q(j)$ is true for all $j \in B_{X Y}(k)$. We subdivide the block $B_{X Y}(k)$ into $K=2^{m^{\prime}-1}$ subblocks of equal width. These subblocks are defined by their central columns $j_{l}$, where $l \in[K]$. Note that their width just fits to the precondition of the next stage because $B_{X Y}(k)$ has width $\frac{N_{s-1}+1}{4}-1$ and, consequently, all $B\left(j_{l}\right)$ have width:

$$
\frac{N_{s-1}+1}{4 \cdot 2^{m^{\prime}-1}}-1=\frac{2^{m-(s-1)\left(m^{\prime}+1\right)}}{4 \cdot 2^{m^{\prime}-1}}-1=\frac{2^{m-(s-1)\left(m^{\prime}+1\right)}}{2^{m^{\prime}+1}}-1=2^{m-s\left(m^{\prime}+1\right)}-1
$$

Due to the conditions encoded in predicate $Q(j)$ for a given color $c=c_{s}$ and column $k$ we make the following case distinction:

Case 1: $\forall_{l \in[K]} \exists_{j^{\prime} \in B\left(j_{l}\right)} c_{s} \in U_{1, j^{\prime}}$

Case 2: $\exists_{l \in[K]} \forall_{j^{\prime} \in B\left(j_{l}\right)} c_{s} \notin U_{1, j^{\prime}}$

In Case 1 we can immediately derive a contradiction using the constructions of Proposition 13: First we horizontally truncate the current tableau $\mathcal{M}$ to the block $B_{X Y}(k)$, then we use a selective truncation with $m^{*}=m^{\prime}$, where the even columns (indexed by $2 l$ for $l \in[K]$ ) of the new tableau are the ones that separate in $\mathcal{M}$ the subblocks $B_{j_{l}}$ and $B_{j_{l+1}}$ from each other and the odd columns (indexed $2 l-1$ ) are chosen from $B_{j_{l}}$ with respect to fulfilling the property $c_{s} \in U_{1, j^{\prime}}$. We show that $c_{s}$ is also unique in the top set of an even column. Supposing that $c=c_{s}$ is not unique in that set contradicts condition (c) of predicate $Q(j)$. Thus $c_{s}$ is unique everywhere in the first row of the new tableau. With respect to condition (b) it does not occur at all in the third row or deeper. Each column of this new tableau $\mathcal{M}^{\prime}$ has depth $d \geq 3$ because all columns of $\mathcal{M}^{\prime}$ have been selected from a quarter subblock $B_{X Y}(k)$. Next we apply a vertical truncation (deletion of top rows) to $\mathcal{M}^{\prime}$ to obtain an $m^{\prime} \times N^{\prime}$ tableau $\mathcal{M}^{*}$. This way at least the two top rows of $\mathcal{M}^{\prime}$ are deleted and thus color $c_{s}$ does not occur anymore in $\mathcal{M}^{*}$. As a result $\mathcal{M}^{*}$ is a $(t-1)$-conform $m^{\prime} \times N^{\prime}$ tableau. 
Case 2 is the easier one because horizontally truncating $\mathcal{M}$ to a block $B\left(j_{l}\right)$ such that $\forall_{j^{\prime} \in B\left(j_{l}\right)} c_{s} \notin U_{1, j^{\prime}}$ yields the precondition for the next stage with $C_{s}=C_{s-1} \cup\left\{c_{s}\right\}$.

\section{Conclusion}

We have shown tight bounds for the chromatic AGP for orthogonal simple polygons if based on r-visibility. While the upper bound proofs use known techniques, we consider our lower bound techniques to be the main technical contribution of the paper. The multicolor tableau technique used for l-visibility can also directly be applied to the r-visibility version of the problem, but does not result in a tight bound, see [8]. Our lower bound technique for r-visibility, however, does not easily generalize to the l-visibility version of the problem, as it relies on the bijection with monotone paths in the spine tree, which does not exist in that case. It would therefore be of interest to combine both techniques to obtain a stronger $\Omega(\log \log n)$ lower bound for $\chi_{c f}^{l}(n)$ as well. We conjecture that this is indeed the lower bound for stretched spike polygons. But one cannot hope for more, since $O(\log \log n)$ is also an upper bound for the conflict-free guarding of stretched spike polygons using line visibility. To improve this lower bound one has to look for other polygons.

\section{References}

1 A. Bärtschi and S. Suri. Conflict-free Chromatic Art Gallery Coverage. Algorithmica 68(1): 265-283, 2014.

2 A. Bärtschi, S.K. Ghosh, M. Mihalak, T. Tschager, and P. Widmayer. Improved bounds for the conflict-free chromatic art gallery problem. In Proc. of 30th Symposium on Computational Geometry, pages 144-153, 2014.

3 V. Chvátal. A combinatorial theorem in plane geometry. Journal of Combinatorial Theory, Series B, 18(1):39-41, 1975.

4 L. H. Erickson und S. M. LaValle. An Art Gallery Approach to Ensuring that Landmarks are Distinguishable, in Proc. Robotics: Science and Systems VII, Los Angeles, pages 81-88, 2011.

5 S. Fisk. A short proof of Chvátal's Watchman Theorem. Journal of Combinatorial Theory, Series B, 24(3):374-374, 1978.

6 S. P. Fekete, S. Friedrichs, and M. Hemmer. Complexity of the General Chromatic Art Gallery Problem. arXiv 1403.2972 [cs.CG], 2014.

7 F. Hoffmann, On the Rectilinear Art Gallery Problem, Proc. 17th ICALP, Springer LNCS 443, 717-728, 1990.

8 F. Hoffmann, K. Kriegel, and M. Willert. Almost Tight Bounds for Conflict-Free Guarding of Orthogonal Art Galleries. arXiv:1412.3984 [cs.CG], 2014.

9 R. Motwani, A. Raghunathan, and H. Saran. Covering orthogonal polygons with star polygons: The perfect graph approach. Comput. Syst. Sci. 40 (1990) 19-48.

10 J. O'Rourke. Art Gallery Theorems and Algorithms. Oxford University Press, New York, NY, 1987.

11 J. Pach and G. Tardos. Coloring axis-parallel rectangles. J. Comb. Theory Ser. A, 117(6):776-782, Aug 2010

12 T. Shermer. Recent results in art galleries (geometry). Proceedings of the IEEE, 80(9): 1383-1399, September 1992

13 D. D. Sleator and R. E. Tarjan. A data structure for dynamic trees. Journal of Computer and System Sciences, 26(3):362-391, 1983. 
14 S. Smorodinski. Conflict-Free Coloring and its Applications. In Geometry - Intuitive, Discrete, and Convex, volume 24 of Bolyai Society Mathematical Studies, Springer Verlag, Berlin 2014

15 J. Urrutia. Art gallery and illumination problems, in J.-R. Sack and J. Urrutia, editors, Handbook on Computational Geometry, pages 973-1026, Elsevier Sc. Publishers, 2000

16 M. Willert. Schranken für eine orthogonale Variante des chromatischen Art Gallery Problems, Bachelor Thesis, FU Berlin, November 2014.

17 C. Worman and M. Keil. Polygon Decomposition and the Orthogonal Art Gallery Problem, Int. J. Comput. Geometry Appl. 17(2), 105-138, 2007 